\title{
Simulación y análisis de un sistema de refrigeración por absorción solar intermitente
}

\author{
Analysis of a Solar Intermittent Absorption Refrigeration System
}

\author{
Diana Julissa Ramírez Álvarez ${ }^{(1)}$, Andrés Felipe Tapia Vertel ${ }^{(2)}$, Valéry José Lancheros Suáres ${ }^{(3)}$, Luis Armando Espitia Sanjuán ${ }^{(4)}$, \\ Yahir Enrique González Dória ${ }^{(5)}$ \\ (1) Estudiante de Ingeniería Mecánica, Universidad de Córdoba. Montería, Colombia. Grupo de investigación en Ingeniería, Ciencia y Tec- \\ nología (ICT).djulissaramirez@correo.unicordoba.edu.co \\ (2) Estudiante de Ingeniería Mecánica, Universidad de Córdoba. Montería, Colombia. Grupo de investigación en Ingeniería, Ciencia y Tec- \\ nología (ICT). aftapia@correo.unicordoba.edu.co \\ (3) MSc en Ingeniería Industrial. Docente de Planta, Programa de Ingeniería Mecánica, Universidad de Córdoba. Montería, Colombia. Grupo \\ de investigación en Ingeniería, Ciencia y Tecnología (ICT). vlanchero@correo.unicordoba.edu.co \\ (4) $\mathrm{PhD}$, MSc en Ingeniería Metalúrgica y de Materiales. Universidad de Córdoba. Montería, Colombia. Grupo de investigación en Ingenie- \\ ría, Ciencia y Tecnología (ICT). luisespitia@correo.unicordoba.edu.co \\ (5) MSc en Ingeniería Industrial. Docente de Planta, Programa de Ingeniería Mecánica, Universidad de Córdoba. Montería, Colombia. Grupo \\ de investigación en Ingeniería, Ciencia y Tecnología (ICT).yahirgonzalez@correo.unicordoba.edu.co
}

Recibido 26 de agosto de 2015. Modificado 20 de noviembre de 2015. Aprobado 30 de noviembre de 2015.

DOI: http://dx.doi.org/10.16924/riua.v0i43.872

\author{
Palabras clave \\ Coeficiente de desempeño, energía solar, refrigeración por \\ absorción, simulación.
}

\section{Resumen}

El departamento de Córdoba cuenta con zonas rurales que no hacen parte del sistema interconectado nacional. Esto crea un problema para sus habitantes ya que, no pueden conservar alimentos. Sin embargo, la alta disponibilidad de radiación solar durante todo el año, ofrece una alternativa para preservar alimentos utilizando equipos de refrigeración por absorción solar. El objetivo de este trabajo fue simular y analizar un sistema de refrigeración por absorción solar, usando el software ASPEN Hysys. Se obtuvo un coeficiente de desempeño (COP) máximo de 1,0 a $93^{\circ} \mathrm{C}$ y $1547 \mathrm{kPa}$, teniendo en cuenta el efecto de distintas variables.

\section{Key words}

Absorption Refrigeration, Coefficient of Performance, Simulation, Solar Energy.

\begin{abstract}
Today, some of the rural areas of Cordoba department are not part of the electrical wiring interconnection system; a problem when it comes to food preservation. Cordoba is subject to high solar radiation throughout the year; therefore, the development of devices powered by an alternative energy source may provide the solution to this problem. The aim of this study was to simulate and analyze an intermittent refrigeration system using ASPEN Hysys software. The simulation was carried out in order to obtain a maximum coefficient of performance (COP) of 1.0 at $93^{\circ} \mathrm{C}$ and $1547 \mathrm{kPa}$, considering the effect of variables such as ammonia concentration, amount of solution, pressure generation, and temperature generation.
\end{abstract}

\section{INTRODUCCIÓN}

El consumo energético global ha presentado un incremento significativo durante los últimos años como resultado del crecimiento acelerado en la población mundial y en la economía, especialmente en los países en desarrollo. De acuerdo con lo anterior, se prevé un incremento del $71 \%$ del consumo energético entre 2003 y 2030 (Sarbu \& Adam, 2011).
Actualmente, la mayor parte de la energía utilizada en todo el mundo proviene principalmente de combustibles fósiles. Estos combustibles son limitados y se utilizan más rápido de lo que se crean, no son amigables con el medio ambiente y su quema se convierte en el principal contribuyente al calentamiento global. Esto traerá escasez con el tiempo. De ahí que sea necesaria la implementación de fuentes de energía amigables con el medio ambiente y que puedan renovarse 
en periodos cortos de tiempo como el sol, el viento, el agua, entre otros.

La energía proveniente del sol es ampliamente utilizada ya que no produce contaminación, es silenciosa y no perjudica el medio ambiente. Un campo viable e importante para la utilización de energía solar está en la refrigeración, debido a la demanda que existe de la misma y la disponibilidad de la radiación solar; por lo tanto, los sistemas que tienen la capacidad de trabajar con este tipo de energía, como los dispositivos de absorción, se presentan como alternativas interesantes de sistemas de refrigeración (Fernández, Brites, Gaspar \& Costa, 2014).

Varias investigaciones han sido realizadas con el objetivo de utilizar la energía solar para producción de frío por medio de dispositivos de refrigeración por absorción intermitente. Francisco et al. (2002) desarrollaron y probaron un sistema de absorción que emplea amoníaco - agua como refrigerante accionado por energía solar para pequeñas áreas rurales. Los resultados mostraron baja eficiencia en el funcionamiento de los dispositivos. Al-Hemiri, Adel \& Ahmed (2010) evaluaron el coeficiente de desempeño para un sistema de refrigeración por absorción mediante el uso de la energía solar directa, usando fracciones de amoníaco acuoso de 0,45 (amoníaco - agua). Fue reportado que la temperatura máxima del generador osciló entre $92^{\circ} \mathrm{C}$ y $97^{\circ} \mathrm{C}$ y la temperatura mínima del evaporador fue de $5^{\circ} \mathrm{C}$ a $10^{\circ} \mathrm{C}$ para el sistema de agua amoniacal. Soukpoen, Yamegueu \& Bassole (2014) describieron las investigaciones y proyectos más significativos para varias condiciones climáticas en África y, recopilaron los logros que se han alcanzado en la utilización de energía solar para la producción de frío. Fue reportado que la mayoría de proyectos se desarrollaron para la conservación de alimentos y vacunas.

Entre los equipos de refrigeración existen los dispositivos por absorción de ciclo intermitente que trabajan con energía térmica y no requieren algún tipo de energía mecánica o eléctrica adicional para su funcionamiento (Said, El-Shaarawi \& Siddiqui, 2012).

El objetivo de este trabajo fue simular y analizar el ciclo de un sistema de refrigeración por absorción solar intermitente para enfriamiento bajo las condiciones climáticas de Córdoba, utilizando una solución compuesta de amoníaco y agua. La simulación se elaboró con el paquete termodinámico Peng- Robinson "P\&R" del software ASPEN Hysys, no solo teniendo en cuenta las características de todos los componentes del sistema, sino también los procesos termodinámicos que ocurren en cada uno de ellos. Adicionalmente, el efecto que tienen algunas variables de proceso en el Coeficiente de desempeño (COP) fue evaluado con el fin de establecer las condiciones de operación que produzcan el máximo COP del sistema.

\section{Materiales y MÉTOdos}

La Figura 1 muestra un esquema simplificado de un sistema de refrigeración que trabaja con energía solar intermitente diseñado para funcionar con una solución compuesta por amoníaco que actúa como absorbedor y agua como absorbente.

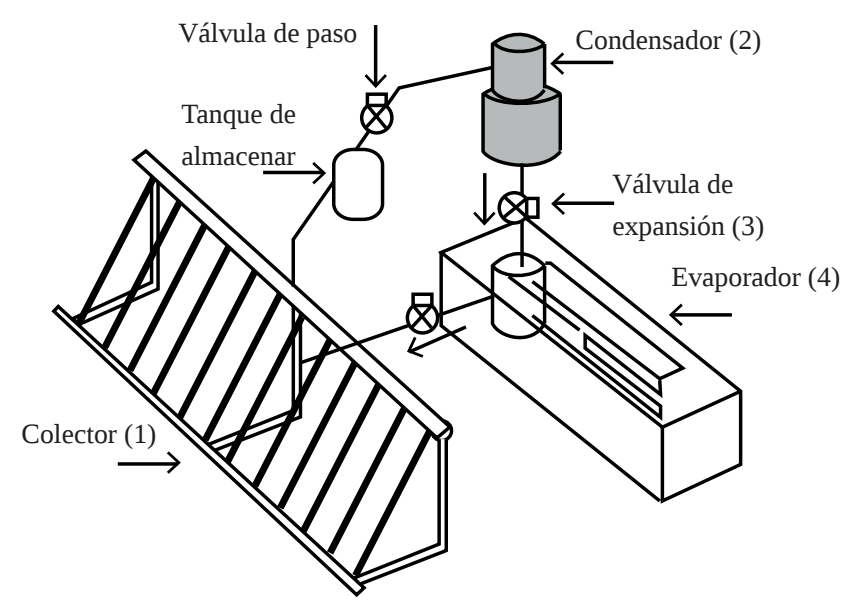

Figura 1. Esquema simplificado de un sistema de refrigeración intermitente que emplea energía solar, diseñado para funcionar con una solución de amoníaco-agua. Fuente: Adaptación de Beltran (2010).

Este sistema es utilizado para realizar la simulación en este trabajo.

El sistema funciona exclusivamente con energía solar y básicamente se compone de un colector (1), un condensador (2), una válvula de expansión (3) y un evaporador (4). El ciclo de refrigeración intermitente se explica a continuación:

Etapa I: el ciclo comienza en el colector (1) con una solución amoníaco-agua conocida como solución fuerte; esta recibe la radiación solar durante el día, ocasionando un incremento en su temperatura y presión, por lo tanto, la energía interna de la solución aumenta y el amoníaco es parcialmente evaporado de la solución. Posteriormente, el amoníaco evaporado se almacena en un tanque con la presión alcanzada en el colector, mientras que, una solución con una concentración de amoníaco menor, conocida como solución débil, permanece en el colector. El vapor de amoníaco almacenado en el tanque actúa como refrigerante. La temperatura y la presión alcanzada en el colector durante el día son denominadas temperatura de generación y presión de generación.

Etapa II: durante la operación nocturna, se abre la válvula de paso ubicada después del tanque de almacenamiento permitiendo el flujo del refrigerante al condensador (2), a la válvula de expansión (3) y al evaporador (4). Los procesos llevados a cabo en los tres componentes mencionados se denominan línea de refrigeración. La temperatura y la presión alcanzada en el colector por la noche se denominan temperatura de absorción y presión de absorción. 
Etapa III: el refrigerante sale del evaporador y regresa al colector donde es absorbido por la solución débil dando lugar a una reacción exotérmica, luego el ciclo inicia nuevamente en la etapa I. Algunas condiciones de operación y características adicionales del sistema de refrigeración intermitente se pueden encontrar en (Chinnappa, 1961,1962; Trombe, Felix, Foex \& Marc 1957).

El ciclo de refrigeración intermitente fue modelado utilizando la ecuación cúbica (EOS) Peng-Robinson (P\&R) del software Aspen Hysys versión 7.2. Este paquete de fluidos ha sido utilizado ampliamente en investigaciones relacionadas con sistemas de refrigeración intermitentes (Darwish et al. 2008; Heidemann \& Rizvi, 1986). En la simulación se tuvieron en cuenta los componentes del sistema, los procesos termodinámicos involucrados en el ciclo y las variables del proceso. Debido a que el software Aspen Hysys no posee algunos de los componentes del ciclo intermitente en su librería, fue necesario descomponer el sistema en subsistemas que sí estuvieran contenidos en las herramientas del software. Cada subsistema recreó todas las características de los componentes del ciclo de refrigeración intermitente. La Tabla 1 muestra los componentes equivalentes utilizados en la simulación y en la Figura 2 se observa el ciclo de refrigeración por absorción intermitente en la interface del software Aspen Hysys.

\begin{tabular}{|c|c|c|}
\hline Etapa & $\begin{array}{l}\text { Componentes } \\
\text { de refrigerador } \\
\text { intermitente }\end{array}$ & Componentes de Hysys \\
\hline 1 & Colector (generador) & $\begin{array}{ll}\text { - } & \text { Bomba } \\
\text { - } & \text { Separador de fases } \\
\end{array}$ \\
\hline \multirow{3}{*}{ II } & Condensador & - $\quad$ Enfriador por aire \\
\hline & Válvula de expansión & - Válvula \\
\hline & Evaporador & - Calentador \\
\hline \multirow{2}{*}{ III } & Colector (tanque) & $\begin{array}{ll}\text { - } & \text { Enfriador por aire } \\
\text { - } & \text { Válvula } \\
\end{array}$ \\
\hline & Colector (absorbedor) & $\begin{array}{ll}\text { - } & \text { Mezclador } \\
\text { - } & \text { enfriador por aire }\end{array}$ \\
\hline
\end{tabular}

Tabla 1. Equivalencia de los componentes del sistema de refrigeración intermitente utilizados en el simulador Aspen Hysys. Fuente: Elaboración propia, 2015.

La simulación se realizó de la siguiente manera:

Etapa I: el incremento en la temperatura y presión de generación se simularon utilizando una bomba (a). Seguidamente, el incremento de temperatura a presión constante fue simulado utilizando un separador de fases (b).

Etapa II: los procesos de disminución de temperatura del refrigerante en el condensador, disminución de presión y temperatura en la válvula de expansión y aumento de temperatura del refrigerante en el evaporador fueron simulados

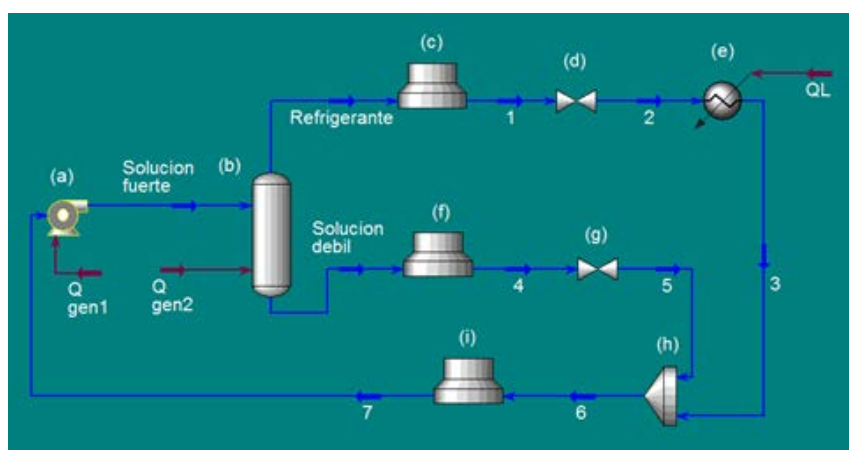

Figura 2. Ciclo de refrigeración intermitente equivalente utilizado en el simulador Aspen Hysys. Fuente: Elaboración propia

con un enfriador de aire (c), una válvula (d) y un calentador (e) respectivamente.

Etapa III: la disminución en la presión y la temperatura de absorción fue simulada utilizando un enfriador de aire (f) y una válvula (g). La reacción exotérmica fue representada con un mezclador (h) y un enfriador de aire (i).

Con el objetivo de encontrar las mejores condiciones de operación del sistema en función de algunas variables de proceso, se llevaron a cabo seis simulaciones. La primera simulación se realizó utilizando las variables mostradas en la Tabla 2, cuyos valores fueron escogidos con base en la literatura de ciclos de refrigeración intermitente. En esta simulación se obtuvo un COP de referencia, calculado con la siguiente ecuación típicamente utilizada por investigadores del área (Darwish, Al-Hashimi \& Al-Mansoori, 2008; Hernandez, Rivera, Colorado \& Moreno-Quintanar, 2012; ElShaarawi, Said \& Siddiqui, 2014, 2015; Marcos, Izquierdo \& Palacios, 2011).

$$
C O P: \frac{Q e}{Q s}
$$

El COP es definido como la relación entre la capacidad que tiene un dispositivo de refrigeración para remover el calor de un lugar que se desea mantener frío (Qe) y la energía suministrada para el funcionamiento (Qs) (Fernández et al., 2014).

Las siguientes cuatro simulaciones se llevaron a cabo utilizando las variables de proceso con mayor influencia sobre el COP teniendo en cuenta los resultados de otras investigaciones (Darwish et al. 2008; Hernández et al. 2012; Marcos et al. 2011). Los investigadores determinaron que el desempeño de un sistema de refrigeración solar es influenciado por la cantidad de refrigerante, presión de generación, temperatura de generación y concentración de amoníaco en la solución.

Estas simulaciones se elaboraron modificando cada una de las variables en un rango, mientras las otras se mantuvieron constantes, con el fin de estudiar el efecto aislado de cada una 
sobre el COP. Los rangos de valores mostrados en la Tabla 3 fueron establecidos acorde a la literatura (Darwish et al. 2008; Hernández et al. 2012; El-Shaarawi 2014). Los resultados de cada simulación se analizaron graficando el COP en función de cada variable de proceso, para escoger el rango de valores que produjeron los valores más altos de COP. En la última simulación, estos valores fueron empleados con el propósito de estudiar el efecto combinado de las variables de proceso sobre el COP.

\begin{tabular}{|c|c|}
\hline Temperatura de condensación & $41^{\circ} \mathrm{C}$ \\
\hline Temperatura de evaporación & $36^{\circ} \mathrm{C}$ \\
\hline Temperatura de generación & $90^{\circ} \mathrm{C}$ \\
\hline Presión de generación & $1379 \mathrm{kPa}$ \\
\hline Caída de presión en la expansión & $150 \mathrm{kPa}$ \\
\hline Temperatura ambiente en el día & $32^{\circ} \mathrm{C}$ \\
\hline Temperatura ambiente en la noche & $24^{\circ} \mathrm{C}$ \\
\hline Concentración de amoníaco en la solución & $54 \% \mathrm{p} / \mathrm{p}$ \\
\hline Operación durante el día & $8 \mathrm{hours}$ \\
\hline Operación durante la noche & $8 \mathrm{hours}$ \\
\hline Cantidad de solución & $7,2 \mathrm{~kg}$ \\
\hline
\end{tabular}

Tabla 2. Variables de proceso utilizadas en la primera simulación para obtener un COP de referencia.

Fuente: Elaboración propia, 2015.

\begin{tabular}{|c|c|}
\hline \multicolumn{2}{|c|}{ Rango de valores para las variables del proceso } \\
\hline Temperatura de generación & $70^{\circ} \mathrm{C}-140^{\circ} \mathrm{C}$ \\
\hline Presión de generación & $1310 \mathrm{Kpa}-1700 \mathrm{kPa}$ \\
\hline Concentración de amoníaco & $42-56 \% \mathrm{p} / \mathrm{p}$ \\
\hline Cantidad de solución & $4 \mathrm{~kg}-8 \mathrm{~kg}$ \\
\hline
\end{tabular}

Tabla 3. Rango de valores para las variables de proceso utilizadas en las simulaciones 2 a 5 .

Fuente: Elaboración propia, 2015.

\section{Resultados y Discusiones}

En la primera simulación se obtuvo un COP de referencia de 0,2856 .

Las figuras 3 y 4 muestran el comportamiento del COP en función de la temperatura de generación, la presión de generación, la cantidad de solución y la concentración de amoníaco en la solución.

Como se puede observar en la Figura 3(a), el COP se incrementa con el aumento de la temperatura, alcanzando un valor máximo de 0,73 a $122,5^{\circ} \mathrm{C}$. A partir de este punto, el COP decrece con el aumento en la temperatura. Durante el proceso de generación, es deseable que el refrigerante esté compuesto solo por amoníaco, sin embargo, durante el ciclo está contaminado por vapor de agua, cuya cantidad varía en función de la temperatura de generación. La presencia de vapor de agua disminuye la capacidad de absorción de calor del refrigerante. A $70^{\circ} \mathrm{C}$ el refrigerante está contaminado con $1 \%$ de vapor de agua. Con el incremento de la temperatura, la cantidad de vapor en el refrigerante aumenta alcanzando un $10 \%$ de concentración a $122,5^{\circ} \mathrm{C}$; sin embargo, la cantidad de amoníaco evaporado también aumenta. Esto incrementa la capacidad del sistema y por consiguiente el COP. No obstante, para temperaturas superiores a $122,5^{\circ} \mathrm{C}$ la cantidad de vapor de agua es demasiada, incrementando la temperatura en el evaporador, por lo tanto, los valores de Qe y COP comienzan a disminuir. La cantidad de vapor de agua a una temperatura de $140^{\circ} \mathrm{C}$ es del $20 \%$. El máximo porcentaje de vapor de agua que se puede permitir en el sistema sin afectar el COP está alrededor del $10 \%$. Un comportamiento similar del COP en función de la temperatura fue reportado por Said et al. (2015).

La Figura 3(b) muestra el comportamiento del COP en función de la presión de generación. En general, los sistemas de refrigeración requieren de una diferencia de presión para que el refrigerante fluya a través de todos los componentes de la línea de refrigeración. Los sistemas de absorción intermitente producen el diferencial de presión porque la presión de generación es mayor a la presión de absorción. Cuando la presión de generación es baja, el diferencial de presión disminuye. Esto trae como consecuencia la disminución del flujo de refrigerante a través del sistema. Este comportamiento es observado en la figura 3(b) para el rango de presión de 1300 a 1380 kPa. Para una presión aproximada de 1400 kPa, el COP se incrementa con el aumento de la presión porque la presión de generación es más alta que la presión de absorción y se mejora el rendimiento del sistema. El COP continuó incrementando con el aumento de la presión, y alcanzó un máximo de 0,8 a $1540 \mathrm{kPa}$. Una vez alcanza este punto, el COP comienza a decrecer con el aumento de la presión. Sin embargo, el incremento en la presión de generación trae consigo efectos negativos sobre el COP. Si la presión de generación es demasiado alta en comparación con la presión de evaporación del amoníaco, la cantidad de amoníaco evaporado disminuye, como se observó a presiones mayores de $1550 \mathrm{kPa}$. Kim, Wang, \& Machielsen (2003) reportaron un comportamiento similar en el COP.

El efecto de la cantidad de solución sobre el COP se muestra en la Figura 4(a). Se puede observar que el COP incrementa de manera constante con el aumento en la cantidad de solución, alcanzando un máximo de 0,31 para una cantidad de 10,4 kg de solución. La cantidad de amoníaco evaporado es mayor si la cantidad de solución es mayor; grandes cantidades de amoníaco evaporado extraen más cantidad de calor en el evaporador. Sin embargo, el efecto de la cantidad de solución fue bajo sobre el COP; un incremento de 2,5 veces en la cantidad de solución solamente aumentó el COP de 0,26 a 0,31 aproximadamente. Si se desea incrementar el COP modificando la cantidad de solución fuerte, es estrictamente 

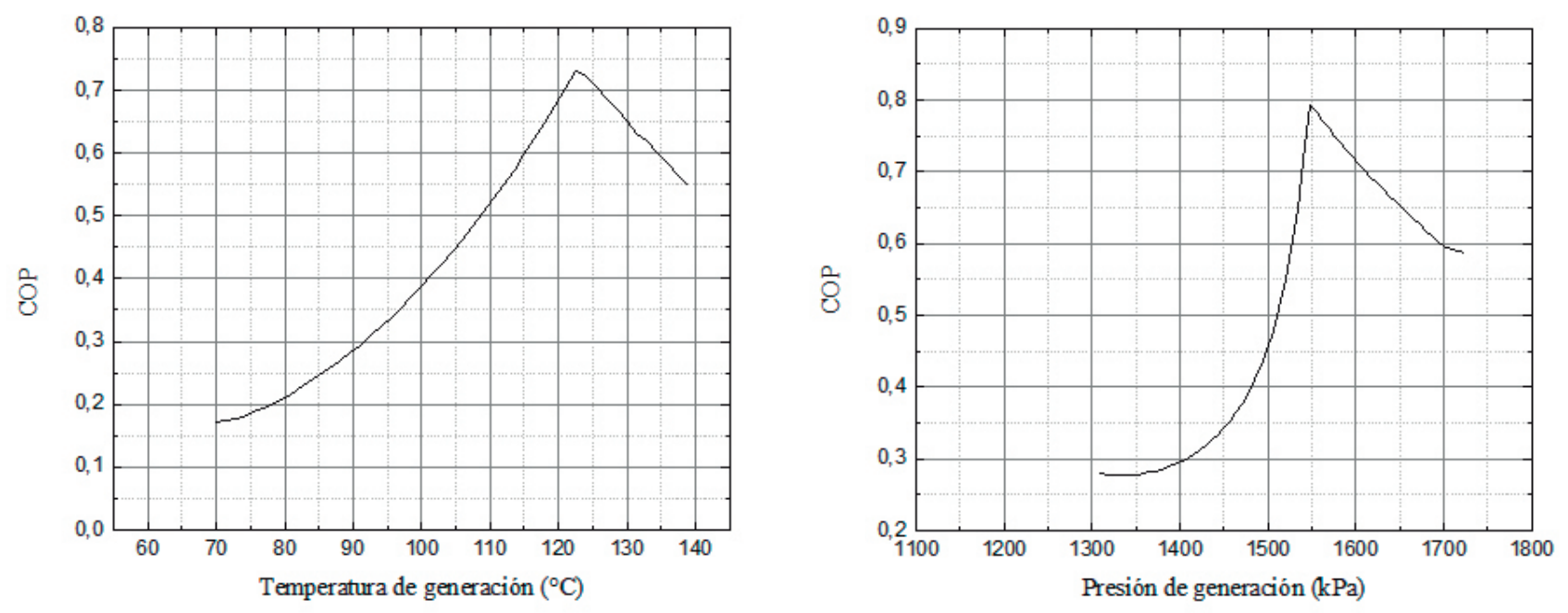

Figura 3. (a) Efecto de la temperatura de generación en el COP, (b) Efecto de la presión de generación en el COP. Fuente: Elaboración propia, 2015.
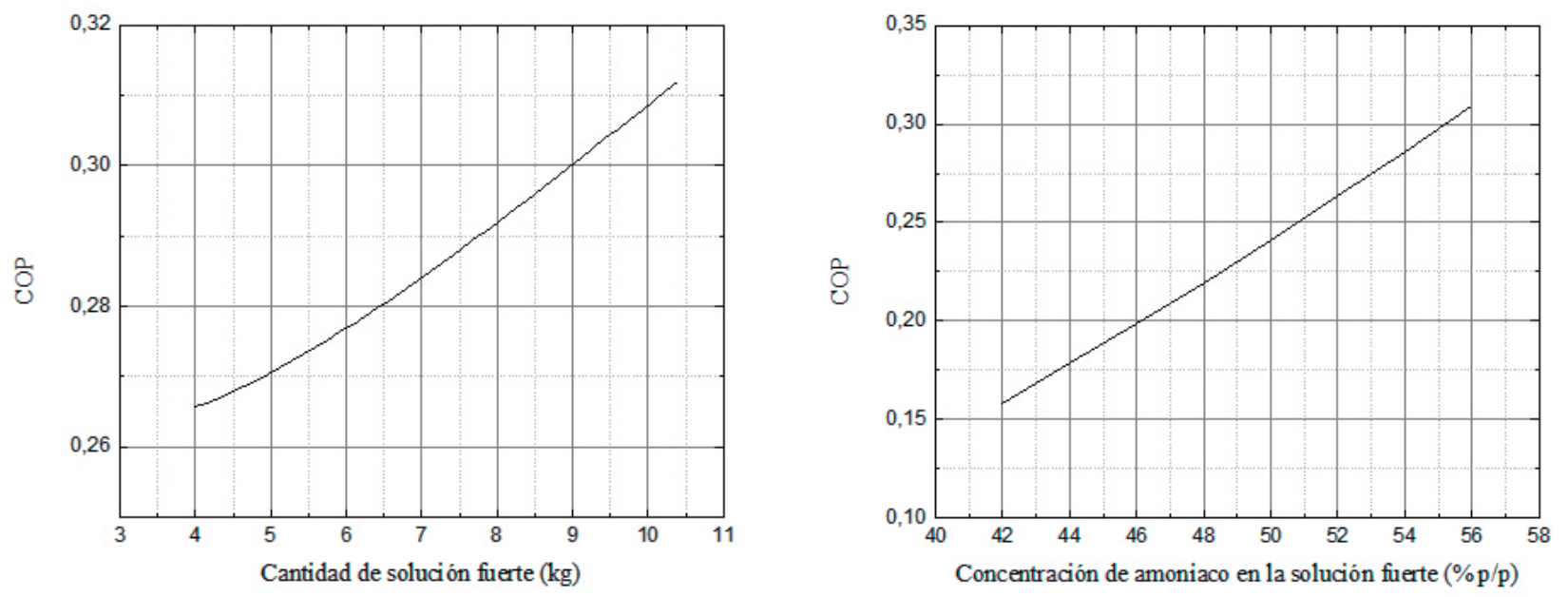

Figura 4. (a) Efecto de la cantidad de solución fuerte en el COP, (b) Efecto de la concentración de amoníaco de la solución fuerte en el COP. Fuente: Elaboración propia, 2015.

necesario incrementar las dimensiones del colector. La Figura 4(b) muestra el COP en función de la concentración de amoníaco en la solución fuerte. Se puede observar que la concentración de amoníaco tuvo un efecto similar al de la cantidad de solución fuerte en el COP, y solamente se incrementó alrededor de 0,16 a 0,31. Estos resultados mostraron que el máximo porcentaje de concentración de amoníaco permitido para estas condiciones fue de $56 \%$. El porcentaje de concentración de amoníaco no puede exceder este valor porque la capacidad de absorción disminuye como consecuencia de la disminución en la cantidad de solución débil. La cantidad de solución débil debe ser suficiente para absorber el refrigerante, de lo contrario, el diferencial de presión disminuye y el refrigerante no circula a través de la línea de refrigeración.
A partir de estos resultados, se concluyó que la cantidad de solución fuerte y la concentración de amoníaco tienen poco efecto sobre el COP. Por esta razón, solo se utilizaron los resultados de la temperatura y la presión de generación en la última simulación. El rango de valores para la temperatura y la presión de generación empleados en la última simulación se seleccionaron con base al COP de referencia obtenido en la primera simulación y a los valores de COP más altos obtenidos en las simulaciones 2 y 3 . En el caso de la temperatura de generación, el valor mínimo del rango correspondiente al COP de referencia fue $90^{\circ} \mathrm{C}$ y el máximo $124^{\circ} \mathrm{C}$. Con respecto a la presión se tomó como valor mínimo 1379 kPa y máximo 1547 kPa. Los valores para la concentración de amoníaco en la solución fuerte y la cantidad de solución fuerte fueron de $56 \%$ p/p y 10,4 kg respectivamente, que corresponden a 
los valores más altos de COP obtenidos en las simulaciones 4 y 5.

La Figura 5 presenta el efecto combinado de la temperatura y de la presión de generación sobre el COP. Se observa que el máximo COP fue de 1,0 a $93^{\circ} \mathrm{C}$ y $1547 \mathrm{kPa}$. En condiciones reales, se considera que en este sistema de absorción solar intermitente no es posible alcanzar este valor, ya que este solo funciona con energía solar y no posee elementos adicionales que ayuden a obtenerlo. Por otra parte, algunos autores han diseñado y construido equipos de refrigeración solar con elementos y mezclas de refrigerantes que mejoran el desempeño de estos sistemas, sin embargo, no han conseguido llegar a un COP de 1,0, como es el caso de Brendel, Zetzsche \& Müller-Steinhagen (2010), quienes construyeron un sistema de absorción de operación continua que empleaba amoníaco y agua, y reportaron un COP de 0,74. Agyenim, Knight \& Rhodes (2010) construyeron un sistema de absorción de operación continua que empleaba LiBr y agua como refrigerante y alcanzaron un COP de 0,58. Ahora con respecto a los sistemas intermitentes que emplean energía solar y una mezcla de amoníaco - agua como refrigerante, El-Shaarawi \& Ramadan (1986) construyeron un dispositivo intermitente y obtuvieron un COP máximo de 0,51 utilizando un rectificador.

Por otro lado, se deben tener en cuenta las condiciones climáticas del departamento de Córdoba, con el fin de realizar un estudio acertado del valor de COP que se pueda obtener en la región. Se tiene conocimiento de que la radiación solar disponible en el departamento de Córdoba es de 5,0 a 5,5 kWh/ $\mathrm{m}^{2}$ (UPME, 2015). Esto permite alcanzar presiones de generación cercanas a $1448 \mathrm{kPa}$, por lo tanto, para esta presión y la temperatura de $93^{\circ} \mathrm{C}$ es posible alcanzar un COP entre 0,45 y 0,5 . Este valor se considera aceptable y alcanzable en la realidad, implementando dispositivos que ayuden a aumentar la temperatura de generación o, en su defecto, aumentar el calor incidente en el equipo y de esta manera mejorar el COP. Esto se logra utilizando componentes sencillos como los colectores parabólicos o de concentración (El Fadar, Mimet \& Perez-Garcia,2009; hidambarama et al. 2011; Sarbu \& Sebarchievici, 2015), y elementos simples como válvulas de control de flujo y de expansión que ayuden a controlar el proceso.

\section{VALIDACIÓN DE LA SIMULACIÓN}

La simulación fue validada por medio de la comparación del COP obtenido bajo las condiciones climáticas del departamento de Córdoba y el COP obtenido a través de investigaciones similares. De acuerdo con Siddiqui y Said (2015), los investigadores El-Shaarawi y Ramadan construyeron un dispositivo intermitente que empleó como mezcla de trabajo amoníaco-agua y utilizaron un rectificador. El máximo COP encontrado fue de 0,51. Los autores reportaron que el rectificador disminuyó el desempeño del sistema porque permitió el paso de vapor de agua hacia la

\section{COP en función de la temperatura y presión de generación}
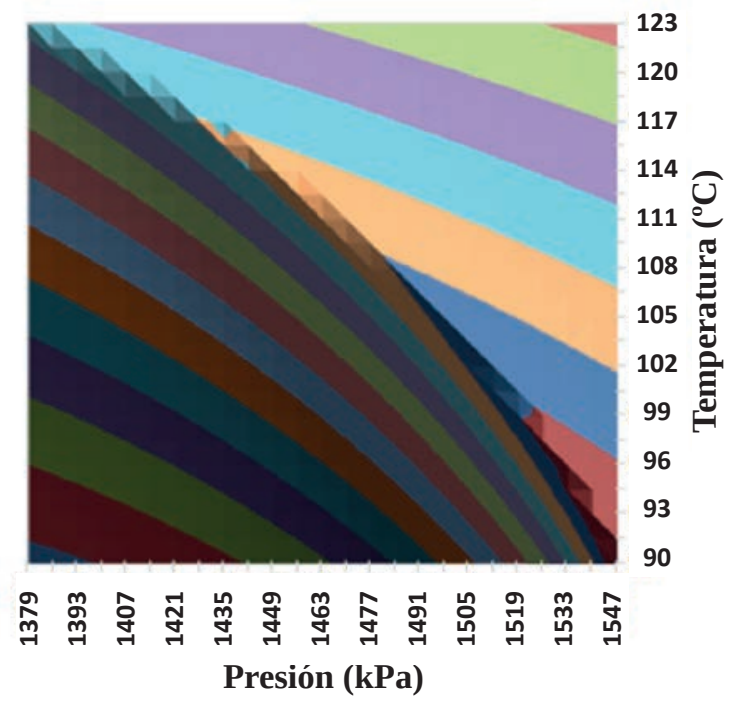

in $0,1-0,15=0,15-0,2=0,2-0,25=0,25-0,3=0,3-0,35$

COP $=0,35-0,4=0,4-0,45=0,45-0,5=0,5-0,55 i=0,55-0,6$

$=0,6-0,65=0,65-0,7^{\prime}=0,7-0,75=0,75-0,8 i=0,8-0,85$

프 $0,85-0,9=0,9-0,95: 0,95-1$

Figura 5. Efecto de la temperatura de generación y de la presión de generación sobre el COP.

Fuente: Elaboración propia

condensadora. Chinnappa (1961) evaluó el desempeño de un refrigerador por absorción intermitente operado por un colector de placas planas y utilizó la mezcla amoníaco-agua como sustancia de trabajo. Experimentalmente obtuvo un COP de 0,25. La Tabla 4 muestra los porcentajes de error obtenidos al comparar el COP de la última simulación con las investigaciones presentadas previamente.

Se debe destacar que en estas simulaciones no se tuvo en cuenta el calor perdido a través de la tubería durante los tiempos de operación del día y la noche. Las pérdidas de calor producen una disminución en la presión de generación causando una reducción en el COP. Por tal motivo, el siguiente paso de esta investigación es la construcción de un sistema de refrigeración intermitente que opere con las condiciones presentadas anteriormente.

\begin{tabular}{|c|c|c|c|}
\hline Investigación & COP & $\begin{array}{c}\text { COP } \\
\text { Simulación }\end{array}$ & \%error \\
\hline El-Shaarawi \& Ramadan (1986) & 0,51 & 0,5 & $2 \%$ \\
\hline Chinnappa (1961) & 0,25 & 0,24 & $4 \%$ \\
\hline
\end{tabular}

Tabla 4. COP de la última simulación y COP reportados en la literatura Fuente: Elaboración propia, 2015 


\section{Conclusiones}

Se logró simular y analizar el ciclo de refrigeración por absorción solar intermitente utilizando las variables más influyentes sobre el COP. Los resultados obtenidos se presentan a continuación:

- Las variables que tuvieron mayor influencia sobre el COP fueron la temperatura de generación y la presión de generación mientras que, las menos influyentes fueron la cantidad de solución fuerte y la concentración de amoníaco en la solución fuerte.

- El máximo COP fue de 1,0 y se obtuvo a $93^{\circ} \mathrm{C}$ y $1547 \mathrm{kPa}$. Sin embargo, bajo condiciones reales no es posible alcanzar este COP, porque el dispositivo es accionado solo con energía solar.

- Para un valor de radiación de 5,0 a 5,5 kWh/m² disponible en el departamento de Córdoba se pueden alcanzar presiones próximas a $1448 \mathrm{kPa}$, por lo tanto, el COP adecuado de operación de un sistema de refrigeración intermitente bajo las condiciones climáticas de Córdoba y las condiciones de operación simuladas fue 0,5 a $93^{\circ} \mathrm{C}$ y $1448 \mathrm{kPa}$. Cabe recordar que se deben utilizar componentes en el sistema que ayuden a aprovechar al máximo la radiación solar y permitan un excelente manejo de los fluidos en la tubería. De esta manera, se evitan fugas u otros inconvenientes en el sistema que puedan llegar a afectar su rendimiento.

\section{REFERENCIAS}

A. De Francisco, R. I. (2002). Development and testing of a prototype of low-power water-ammonia adsorption equipment for solar energy applications. Renewable Energy 25(4), 537-544. doi:10.1016/S0960-1481(01)00093-3

A. El Fadar, A. M.-G. (2009). Study of an adsorption refrigeration system powered by parabolic trough collector and coupled with a heat pipe. Renewable Energy, 34(10), 2271-2279. doi:10.1016/j.renene.2009.03.009

Adel A. A, -H. \&. (2010). The use of direct solar energy in absorption refrigeration employing nh3 - h2o system. Iraqi Journal of Chemical and Petroleum Engineering, 13-21.

Agyenim F, K. I. (2010). Design and experimental testing of the performance of an outdoor $\mathrm{LiBr} / \mathrm{H} 2 \mathrm{O}$ solar thermal absorption cooling system with a cold store. Sol Energy 84(5),735-44. doi:10.1016/j.solener.2010.01.013

Beltrán, R. G. (2010). Refrigerador solar - ciclo de absorción intermitente. Bogota: Universidad de los Andes.

Brendel T, Z. M.-S. (2010). Ninth IIR Gustav Lorentzen conference. Sidney.

B. S. (2014, june). bp. Retrieved from: http://www.bp.com/ en/global/corporate/about-bp.html
Chidambarama, L.A., Ramana A. S., Kamaraj, G. \& Velraj, R. (2011). Review of solar cooling methods and thermal storage options. Renewable and Sustainable Energy Reviews 15(6), 3220-3228. doi:10.1016/j.rser.2011.04.018

Chinnappa, J. (1961). Experimental study of the intermittent vapour absorption refrigeration cycle employing the refrigerant-absorbent systems of ammonia water and ammonia lithium nitrate. Solar Energy 5(1) 1-18. doi:10.1016/0038-092X(61)90029-9

Chinnappa, J. (1962). Performance of an Intermittent Refrigerator Operated by a Flat-Plate Collector. Solar Energy 6(4), 143-150. doi:10.1016/0038-092X(62)90125-1

Darwish, N., Al-Hashimi, S., \& Al-Mansoori, A. (2008). Performance analysis and evaluation of a commercial absorption-refrigeration water-ammonia (ARWA) system. International Journal of Refrigeration 31(7), 1214-1223. doi:10.1016/j.ijrefrig.2008.02.005

El-Shaarawi , M., Said, S., \& Siddiqui, F. (2014). Unsteady thermodynamic analysis for a solar driven dual storage absorption refrigeration cycle in Saudi Arabia. Solar Energy 110, 286-302. doi:10.1016/j.solener.2014.08.032

El-Shaarawi, M., \& Ramadan, R. (1986). Solar refrigeration inthe Egyptian climate. Solar energy 37(5), 347-361. doi:10.1016/0038-092X(86)90131-3

Fernandes, M. S., Brites, G. J. V. N., Costa, J. J., Gaspar, A. R. \& Costa, V. A. F. (2014). Review and future trends of solar adsorption refrigeration systems. Renewable and Sustainable Energy Reviews 39, 102-123. doi:10.1016/j. rser.2014.07.081

Heidemann, R., \& Rizvi, S. (1986). Correlation of ammonia-water equilibrium data with various modified PengRobinson equations of state. Fluid Phase Equilibria 29, 439-446. doi:10.1016/0378-3812(86)85042-7

Hernandez, J., Rivera, W., Colorado, D., \& Moreno-Quintanar, G. (2012). Optimal COP prediction of a solar intermittent refrigeration system for ice production by means of direct and inverse artificial neural networks. Solar Energy 86(4), 1108-1117. doi:10.1016/j.solener.2011.12.021

I. Sarbu, C. S. (2015). General review of solar-powered closed sorption refrigeration systems. Energy conversion and management 105, 403-422. doi:10.1016/j.enconman.2015.07.084

I. Sarbu, M. A. (2011). Applications of solar energy for domestic hot-water and buildings heating/cooling. International Journal of Energy 5(2), 34-42.

Kim, D.-S., Wang, L., \& Machielsen, C. H. (2003). Dynamic modelling of a small-scale nh3/h2o absorption chiller. 21st IIR international congress of refrigeration: "Serving 
the needs of mankind", (pp. 1-8). Paris: Institut International du Froid.

Kokouvi Edem N'Tsoukpoen, D. Y. (2014). Solar sorption refrigeration in Africa. Renewable and Sustainable Energy Review 35, 318-335. doi:10.1016/j.rser.2014.04.030

Marcos, J., Izquierdo, M., \& Palacios, E. (2011). New method for COP optimization in water- and air-cooled single and double effect LiBr-water absorption machines. International Journal of Refrigeration, 34(6), 1348-1359. doi:10.1016/j.ijrefrig.2011.03.017

Mohand Berdja, B. A. (2014). Design and realization of a solar adsorption refrigeration machine powered by solar energy. Energy Procedia 48, 1226 - 1235. doi:10.1016/j. egypro.2014.02.139

Rasul, M., \& Murphy, A. (2006.). Solar powered intermittent absorption refrigeration unit. Australasian Power Engineering Conference AUPEC.

Said, S.A.M., El-Shaarawi, M. A. I. \& Siddiqui, M.U. (2013). Intermittent absorption refrigeration system equipped with an economizer. Energy 61, 332-344. doi:10.1016/j. energy.2013.09.034

Said, S.A.M., El-Shaarawi, M.A.I. \& Siddiqui, M.U. I(2012). Alternative designs for a 24-h operating solar-powered absorption refrigeration technology. International Journal of Refrigeration 97, 1967-1977. doi:10.1016/j.ijrefrig.2012.06.008

Said, S., El-Shaarawi, M., \& Siddiq, M. (2015). Analysis of a solar powered absorption system. Energy Conversion and Management 97, 243-252. doi:10.1016/j.enconman.2015.03.046

Siddiqui, M.U. \& Said, S. A. M. (2015). A review of solar powered absorption system. Renewable and Sustainable Energy Reviews 42, 93-115. doi:10.1016/j. rser.2014.10.014

Trombe, F., \& Foex, M. (1957). The production of cold by means of solar radiation. Solar Energy 1(1), 51-52. doi:10.1016/0038-092X(57)90055-5

Unidad de Planeación Minero Energética (UPME). (2015). Retrieved from: http://www1.upme.gov.co/

W. Rivera, G. M.-Q. (2011). Evaluation of a solar intermittent refrigeration system for ice production operating with ammonia/lithium nitrate. Solar Energy 85(1), 38-45. doi:10.1016/j.solener.2010.11.007

Yunus A. Cengel, M. A. (n.d.). Thermodynamics An Engeenering Approach . In M. A. Yunus A. Cengel, Thermodynamics: An Engineering Approach, 5th edition (pp. 607-637). 